\title{
RENORMALIZATION GROUP APPROACH TO THE INTERACTING BOSE FLUID
}

\author{
F.W. WIEGEL \\ Department of Applied Physics, Twente University of Technology, Enschede, The Netherlands
}

Received 6 October 1977

It is pointed out that the method of functional integration provides a very convenient starting point for the renormalization group approach to the interacting Bose gas. Using such methods we show in a general and non-perturbative way that the critical exponents of the Bose gas are identical to those of a classical system of spins with two real components.

\section{Introduction}

It has been conjectured by several authors that the critical exponents of a quantum system should be identical to those of an appropriately chosen classical system. This conjecture was inspired by the observation that the critical behavior of a system is dominated by the cooperative action of very large numbers of particles. On this macroscopic level quantization supposedly plays no role.

This heuristic argument should be considered as unsatisfactory. The phase transition in the interacting Bose gas, for example, is of an entirely quantummechanical nature because the transition is caused by constructive interference of the de Broglie waves of the particles. When the thermal wavelength of the particles becomes comparable to the interparticle distance the $\lambda$-transition sets in. As the $\lambda$-line consists of critical points there is, therefore, no reason to expect the critical exponents to be independent of Planck's constant. Clearly this conjecture is in need of a proof rather than a heuristic argument.

The first such proof is due to Singh ${ }^{1-4}$ ). This author applied the renormalization group approach to the standard perturbation series for the Bose fluid and calculated the critical exponents to first order in $\epsilon=4-d$, where $d$ is the dimension of space. To this order the exponents are identical to those of a $d$-dimensional two-component Ising model.

In this paper we want to provide a general, non-perturbative proof of the classical nature of the critical exponents. It will be shown that this fact is due 
to a peculiar type of interference of complex contributions to the partition function, which is such that all the non-classical contributions cancel each other by destructive interference upon repeated application of the renormalization group transformation. Our method generalizes Singh's work to all orders in $\epsilon$ and, hopefully, leads to a somewhat simpler proof of the conjecture. The reader might also want to compare recent lectures by the author, which contain an alternative proof based on an explicit calculation of the infinitesimal generator of the renormalization group ${ }^{5}$ ).

\section{Renormalization group theory}

As the method of functional integration forms the subject of a recent review paper ${ }^{6}$ ) there is no need to discuss this formalism here. We only quote one of the rigorous results of that approach:

$$
Z(z, \beta, \Omega)=C\langle\exp \{-F[\phi]\}\rangle_{W} .
$$

Here $Z$ denotes the grand canonical partition function of spinless bosons in a volume $\Omega ; \beta=\left(k_{\mathrm{B}} T\right)^{-1}$ and $C$ is a constant the value of which is not needed here. The average is taken over complex valued (c-number) fields $\phi(r, \theta)$ which are functions of the position in space $(\boldsymbol{r})$ and an additional dimensionless variable $\theta \in[0,1]$. The complex valued weight of a function $\phi$ is given by:

$$
W[\phi]=N^{-1} \exp \left\{-\int_{\Omega} \mathrm{d}^{d} \boldsymbol{r} \int_{\Omega}^{1} \mathrm{~d} \theta\left[\frac{1}{2}|\nabla \phi|^{2}+b \phi^{*} \frac{\partial \phi}{\partial \theta}\right]\right\},
$$

where the normalization constant $N$ must be chosen in such a way that the weight of the whole function space equals unity. Finally, one has

$$
b=m / \beta \hbar^{2}
$$

and

$$
\begin{aligned}
F[\phi]= & -\frac{\mu m}{\hbar^{2}} \int|\phi(\boldsymbol{r}, \boldsymbol{\theta})|^{2} \mathrm{~d}^{d} \boldsymbol{r} \mathrm{d} \theta \\
& +\frac{m^{2}}{2 \beta \hbar^{4}} \int \mathrm{d}^{d} \boldsymbol{r} \mathrm{d} \theta \int \mathrm{d}^{d} \boldsymbol{r}^{\prime} \mathrm{d} \theta^{\prime}|\phi(\boldsymbol{r}, \theta)|^{2} V\left(\boldsymbol{r}-\boldsymbol{r}^{\prime}\right)\left|\phi\left(\boldsymbol{r}^{\prime}, \boldsymbol{\theta}^{\prime}\right)\right|^{2},
\end{aligned}
$$

where $m$ equals the mass of the particles, $\mu$ the chemical potential and $V$ the pair interaction. The various derivations of (1)-(4) have been reviewed in refs. 5 and 6 to which papers we also refer for some minor notational details. The effect of the Bose statistics is represented by the term $b \phi^{*} \partial \phi / \partial \theta$ on the right-hand side of (2); this term is purely imaginary and causes interference of the various complex contributions to the average (1).

The essential step of the renormalization group approach to the evaluation 
of the average (1) is to write

$$
\phi(r, \theta)=\phi_{1}(r, \theta)+\phi_{s}(r, \theta),
$$

where $\phi_{1}$ contains the plane wave components of $\phi$ with a long wavelength and $\phi_{\mathrm{s}}$ those with a short wavelength. The next four steps are as follows.

(a) Perform the average over $\phi_{\mathrm{s}}$ for fixed $\phi_{\mathrm{l}}$.

(b) Introduce new wave vectors $\tilde{k}$ which are related to the old wave vectors $\boldsymbol{k}$ by:

$$
\tilde{\boldsymbol{k}}=\alpha \boldsymbol{k}
$$

where $\alpha>1$ is chosen in such a way that the cut-off in $\tilde{k}$ space equals the original cut-off in $\boldsymbol{k}$ space.

(c) Introduce new position vectors by

$$
\tilde{\boldsymbol{r}}=\alpha^{-1} \boldsymbol{r} .
$$

This implies that $\boldsymbol{k} \cdot \boldsymbol{r}=\tilde{\boldsymbol{k}} \cdot \tilde{\boldsymbol{r}}$ is invariant.

(d) Introduce the new complex field $\tilde{\phi}$ by:

$$
\tilde{\phi}=\alpha^{(d / 2-1)} \phi_{1} \text {. }
$$

As a result of these transformations the partition function reads

$$
Z(z, \beta, \Omega)=\tilde{C}\langle\exp \{-\tilde{F}[\tilde{\phi}]\}\rangle_{\tilde{w}},
$$

where the renormalized weight function is given by

with

$$
\tilde{W}[\tilde{\phi}]=\tilde{N}^{-1} \exp \left\{-\int_{\tilde{\Omega}} \mathrm{d}^{d} \tilde{\boldsymbol{r}} \int_{0}^{1} \mathrm{~d} \theta\left[\frac{1}{2}|\tilde{\nabla} \tilde{\phi}|^{2}+\tilde{b} \tilde{\phi}^{*} \frac{\partial \tilde{\phi}}{\partial \theta}\right]\right\},
$$

$$
\tilde{b}=\alpha^{2} b
$$

Note that $\alpha^{2}>1$, so that $b$ increases under the action of the renormalization group transformation.

In spectral form one writes:

$$
\begin{aligned}
\phi(r, \theta) & =\Omega^{-1 / 2} \sum_{k} \sum_{l=-\infty}^{+\infty} C_{k, l} \exp \{\mathrm{i}(\boldsymbol{k} \cdot \boldsymbol{r}-2 \pi l \theta)\}, \\
W[\phi] & =\prod_{k} \prod_{l=-\infty}^{+\infty} \frac{1}{\pi}\left(\frac{1}{2}|\boldsymbol{k}|^{2}-2 \pi l b \mathrm{i}\right) \exp \left\{-\left(\frac{1}{2}|\boldsymbol{k}|^{2}-2 \pi l b \mathrm{i}\right)\left|C_{k, l}\right|^{2}\right\} \\
& \equiv \prod_{k} \prod_{l=-\infty}^{+\infty} w\left(C_{k, l}\right) .
\end{aligned}
$$

Indefinite repetition of the renormalization procedure will let $b$ tend to infinity. But, as

$$
\begin{aligned}
\lim _{b \rightarrow+\infty} w\left(C_{k, l}\right) & =\frac{1}{\pi}\left(\frac{1}{2}|k|^{2}\right) \exp \left\{-\frac{1}{2}|k|^{2}\left|C_{k, 0}\right|^{2}\right\}, & & \text { if } l=0, \\
& =\delta\left(\operatorname{Re} C_{k, l}\right) \delta\left(\operatorname{Im} C_{k, l}\right), & & \text { if } l \neq 0,
\end{aligned}
$$


the only random fields which contribute in this limit to the renormalized partition function are those fields $\phi(r)$ which do not depend on $\theta$. The weight of such fields is proportional to

$$
\exp \left\{-\int_{\Omega} \mathrm{d}^{d} \boldsymbol{r} \frac{1}{2}|\nabla \phi(\boldsymbol{r})|^{2}\right\},
$$

i.e. the same as the weight functional of a classical system of spins with two real components. As the critical exponents are determined by the way in which the partition function transforms under further application of the operations (a)-(d) the critical exponents will have the same values as those of a system of classical spins with two components.

\section{Conclusions}

It should of course be kept in mind that eq. (1) holds in the grand-canonical ensemble whereas the classical spin model is usually discussed in the canonical ensemble. Hence, a further elimination of the chemical potential is necessary to obtain the critical exponents of the Bose fluid in the canonical ensemble.

A trivial illustration of the different roles of the ensemble in both systems is the following. A glance at (4) shows that a critical value of the temperature can be determined for every choice of $\mu$. Hence, the $\lambda$-line is a line of critical points, in contrast with the classical spin system which has an isolated critical point only.

Finally, it should be kept in mind that our proof holds for bosons only. because the representation defined by (1)-(4) holds for bosons only. The critical exponents of systems of interacting fermions have not yet been determined with the renormalization group.

\section{References}

1) K.K. Singh, Phys. Lett. 51 A (1975) 27.

2) K.K. Singh, Phys. Rev. B12 (1975) 2819.

3) K.K. Singh, Phys. Rev. B13 (1976) 3192.

4) K.K. Singh. Phys. Lett. 57A (1976) 309.

5) F.W. Wiegel, The Interacting Bose Fluid: Path Integral Representations and Renormalization Group Approach, Proc. NATO Advanced Study Institute on Path Integrals, Antwerp, 1977 (Plenum Press, New York, 1978).

6) F.W. Wiegel, Phys. Rep. 16 (1975) 57. 